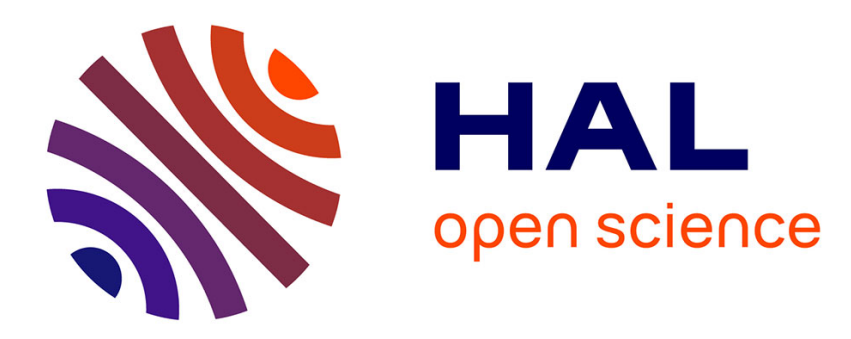

\title{
Écrire le qualitatif : écriture réflexive, écriture plurielle, écriture performance \\ Jean-Luc Moriceau
}

\section{To cite this version:}

Jean-Luc Moriceau. Écrire le qualitatif: écriture réflexive, écriture plurielle, écriture performance. Revue internationale de psychosociologie et de gestion des comportements organisationnels, 2018, 2018-05-14, XXIV (57), pp.45 - 67. 10.3917/rips1.057.0045 . hal-01793350

\section{HAL Id: hal-01793350 https://hal.science/hal-01793350}

Submitted on 16 May 2018

HAL is a multi-disciplinary open access archive for the deposit and dissemination of scientific research documents, whether they are published or not. The documents may come from teaching and research institutions in France or abroad, or from public or private research centers.
L'archive ouverte pluridisciplinaire HAL, est destinée au dépôt et à la diffusion de documents scientifiques de niveau recherche, publiés ou non, émanant des établissements d'enseignement et de recherche français ou étrangers, des laboratoires publics ou privés. 
Fichier auteur. Une version plus avancée a été publié à :

Jean-Luc Moriceau, «Écrire le qualitatif : écriture réflexive, écriture plurielle, écriture performance », Revue internationale de psychosociologie et de gestion des comportements organisationnels 2018/57 (Vol. XXIV), p. 45-67.

\section{ÉCRIRE LE QUALITATIF : Écriture réflexive, écriture plurielle, écriture performance}

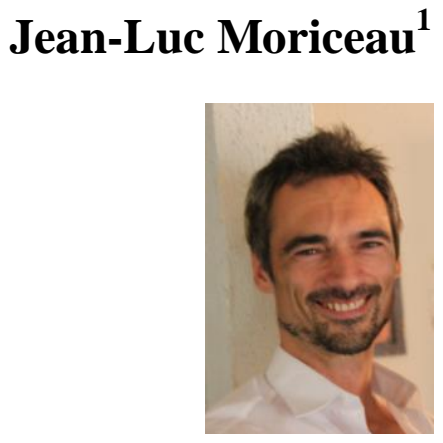

La recherche qualitative ne se formule pas en nombres, fonctions ou tableaux. Avant tout elle se forme et s'exprime dans la texture et l'épaisseur de la langue. Elle est de part en part écriture, et en son cœur, la description est aussi affaire d'expressions et d'impressions, de mots, de formules et de compositions. Écrire et décrire le qualitatif demandent d'inventer une écriture propre, un travail de création tout comme l'artisan façonne son art ou l'acteur travaille sa présence et sa voix. L'écriture n'y est pas véhicule mais création de sens (MARTINET, 2006). La description, originalité et richesse de la méthode qualitative (DUMEZ, 2010), n’y est pas une collection de données mais immersion dans un monde.

Et pourtant, paradoxalement, dans le monde académique, l'écriture est de plus en plus considérée comme seconde et secondaire. Seconde comme s'il existait une pensée pure, non encore contaminée par les mots, après laquelle l'écriture courrait toujours, tentant toujours imparfaitement de la restituer ou la reconstituer. Seconde aussi car elle interviendrait à la fin, une fois la recherche faite, compte-rendu ou rapport, pour communiquer ce qui a été auparavant conçu et réfléchi. Et secondaire, dévalorisée par ceux-là mêmes dont la valeur est évaluée sur leurs écrits.

Et pourtant, second paradoxe, les chercheurs, qui ont fait de longues études, lu beaucoup de textes, qui font profession de manier la langue, s'obligent de plus en plus à utiliser un langage simplifié, raboté, formaté, s'interdisant toutes les possibilités de sens et d'effets - pressés qu'ils sont à produire des articles dans des revues sélectionnées. Dans une envahissante majorité la recherche se limite à une écriture de bois, impersonnelle et abstraite (DANE, 2011, SWORD, 2012, LAROCHE, 2017). La recherche qualitative a donné naissance à de magnifiques ouvrages, des textes de grands mérites académique et littéraire, mais l'écriture qualitative aurait de plus en plus de mal à s'immiscer dans les cadres contemporains. Elle a le désavantage comparatif de ne pas s'encapsuler dans un abstract ou un tableau de résultat (RICHARDSON, 2000). Elle demande du temps et de l'entraînement pour être composée et pour être lue.

Alors souvent l'écriture s'appauvrit, nous perdons un savoir-faire qui se transmettait d'une génération à l'autre. Voici que trop souvent :

« Nous cultivons et nous enseignons un style d'écriture du non-style qui valorise la métaphore limitée, la simplicité et une précision formelle, sinon mathématique. Une grande partie de notre écriture est nettoyée par une bonne douche de dite objectivité, puisque de nombreux auteurs (et lecteurs) du champ considèrent les plaisirs et formes artistes comme interférant avec la présentation de ce qui existe réellement dans un monde social donné. »(VAN MAANEN, 1995, p. 134)

Nombreux sont ceux toutefois qui n'ont pas renoncé. Qui nous offrent des textes à l'écriture immersive, captivante, touchante, nuancée, non comme ornement mais comme intention, comme force poétique pour dire le complexe, le territorialisé, le multiple, comme échappée hors des enfermements, des distributions iniques ou

\footnotetext{
${ }^{1}$ Jean-Luc Moriceau est professeur de méthodes de recherche et de contrôle à l'Institut Mines-Télécom/TEM. Il y est responsable des séminaires pour doctorants et co-animateur de l'équipe ETHOS au sein du LITEM. Ses recherches mettent en avant les affects, l'esthétique, les performances et le sens. Il co-publie en 2018 S'exposer, cheminer, réfléchir : Composer sa méthode de recherche qualitative (EMS), L'art du sens dans les organisations (Eska) et Turn to Film (Sense).
} 
infertiles des places, comme invention d'une voix, comme relai d'autres voix, créations de mondes, voyages dans une sensibilité, évocation d'une condition ou d'une conviction, témoignage, acte thérapeutique, alerte, cri. Et qui nous offrent des descriptions denses, acérées, parlantes, ajustées, détaillées ou ajourées, toutes en reflets et en réflexions. Ceux-là prennent l'écriture et la description comme problème et comme ressource, comme art et comme technique, comme apprentissage et comme expérience pour dire le qualitatif. Pour eux, l'écriture n'interfère pas avec l'accès au réel, elle est l'accès au réel, et elle est tout autant le lieu où ce réel se réfléchit. Et, pour eux, emmener dans l'écriture de nouvelles dimensions, intensités et frontières du réel - affects, espaces, pratiques, matérialités, introspections, traces mémorielles, expériences esthétiques, etc. - c'est enrichir la réflexion sur les organisations, la provoquer, lui donner de nouveaux départs et nouveaux échos.

Toutefois, si l'écriture est accès au réel, si elle est tant constitutive du sens, si elle est théorie (VAN MAANEN, 1995), alors l'écriture ne saurait être la simple expression spontanée d'un auteur. Elle emporte avec elle de nombreux problèmes théoriques et épistémologiques, éthiques et politiques, qu'elle révèle ou qu'elle constitue, et qu'il nous faut réfléchir. Écriture et description se donnent à la fois comme promesses et comme problèmes : comme difficultés qui ouvrent des possibilités, comme possibilités qui engendrent des difficultés. Qui appellent en tout cas réflexivité.

Nombre de ces problématiques apparaissent en écrivant. L'écriture est moins mise en mots d'une réflexion que réflexion elle-même. Elle nous entraîne, nous auteurs et lecteurs, vers des compréhensions et des pensées que nous n'attendions pas, souvent différentes d'un texte à l'autre. C'est quelques-unes de ces problématiques et réflexions qui viennent avec l'écriture, de par ses contraintes et ses potentialités, que nous voudrions présenter ci-après.

Nous soulignerons que l'écriture qualitative est un travail du texte qui est en même temps exercice de réflexivité et d'approfondissement théorique. Nous montrerons certaines évolutions de l'écriture qualitative, comment celle-ci s'éloigne des pièges de la représentation, élargit son champ et pense sa politique. Nous insisterons sur l'enjeu de la voix à trouver, et comment celle-ci invite à des écritures plurielles, moins autoritaires, par exemple féminines, postcoloniales ou dialogiques. Nous verrons aussi les recherches d'une écriture performative, qui met au travail le lecteur, bouscule son assurance de savoir, met en mouvement ses positions et en même temps mesure ses effets vis-à-vis du vulnérable et du fragile, se fait parfois thérapeutique. Enfin, nous conclurons avec l'invitation à penser l'écriture non comme un reflet mais plutôt comme une action, un engagement, une résistance à la prolétarisation, invitation, en reprenant les mots de LETICHE et LIGHTFOOT (2014), à écrire comme si écrire pouvait faire une différence.

\section{L'ÉCRITURE RÉFLEXIVE}

Écrire c'est travailler le texte et travailler la réflexion. L'écriture fait advenir la pensée, elle actualise un virtuel, elle est création. Elle est une fabrique du sens : «comment pourrais-je savoir ce que je pense tant que je ne l'ai pas écrit? » pourrait-on dire en paraphrasant WEICK (1995). Sans ce travail, nous en restons à un magma d'impressions et d'intuitions. Pierce est encore plus net lorsqu'il affirme que sans l'aide d'un encrier, il serait incapable de s'exprimer, même ne lui viendrait pas la moindre idée (ROSSET, apud LAMBERT (2007)). Nous avons tous probablement fait cette expérience de partir avec une idée vague, que nous croyons ferme mais qui se dérobe au moment de la fixer. Un effort d'écriture est alors nécessaire pour la former. Et voici que durant ce travail elle se transforme, rencontre d'autres pensées, se croise avec d'autres textes, s'écarte de l'intuition de départ, se fortifie et nous emmène vers ce que nous trouvons finalement plus satisfaisant. Mais ceci n'est pas l'aboutissement. Cette idée sera repensée plus tard, reterritorialisée dans un texte plus complet. Un texte a sa propre économie, l'idée s'y trouvera dans une écologie, va devoir s'ajouter à l'effort d'expression un effort de composition. Le nouvel agencement pose de nouvelles questions, forçant à de nouvelles réflexions. Écoutons à nouveau Van Maanen à propos de cet indispensable travail du texte :

«Le travail du texte implique des choix, innombrables, concernant la voix, la présence (ou l'absence) de l'auteur, les analogies et les métaphores, les allusions, le jargon professionnel, les images évoquées, le parcours interprétatif, le ton, l'accent porté sur l'empirique ou le théorique, les prétentions à la validité (ou leur absence), les figures de style, etc. » (VAN MAANEN, 2011, p.159)

Rechercher l'écriture juste fait partie de la recherche. Puisqu'en dépendent son sens et son efficacité, mais aussi du fait de toutes les questions qui nous sont ainsi posées. Bien sûr il y a des formats imposés (nous y reviendrons) mais, jusqu'à un certain point, ils n'empêchent pas totalement un espace de jeu, de liberté et de créativité. Ce qui est en jeu n'est pas seulement la question du format, joue aussi notre rapport à l'écriture. Barthes distingue les écrivants des écrivains. Les premiers ne se soucient pas de l'écriture, pour eux la parole n'est qu'un moyen, un instrument de la communication, un véhicule de la pensée. Les seconds travaillent leurs écritures, et ce faisant interrogent le monde. En se demandant comment le dire, toujours inassurés de leur choix, ils interrogent et expérimentent notre façon de le penser. Ils ne prétendent pas dire le tout de la réalité ou de la vérité, mais travaillent leur texte à la recherche d'une certaine forme de justesse, car ils savent que faisons aussi 
le monde, collectivement, avec des mots. Ce qui fait oser à LAMBERT (2007), dans son invitation à penser mais aussi à goûter l'écriture en recherche, un diagnostic un peu différent :

« Nous avons abandonné l'écriture aux marchands. Nous avons accepté que son usage soit industrialisé. Aurions-nous oublié le sujet du langage? Aurions-nous oublié le droit d'écrire hors de la langue du pouvoir? Je dis non et trois fois non, non, non je dis qu'il faut jouer avec les mots pour donner à la pensée, celle de celui qui écrit et celui qui lit, des doutes, des surprises. Verrait-on ici poindre de la vanité qu'il faudrait immédiatement s'en défaire. L'écrivain est un charlatan, et lui sait le dire. L'écrivant un exécutant qui ne dit pas toujours pour qui sa plume tranche, ni quelles blessures son exécution lui laissent. »(LAMBERT, 2007, p. 31).

Au lieu d'interférer dans notre contact avec les organisations, le travail de l'écriture prend au contraire au sérieux ce contact. Il interroge ce contact à partir duquel nous parlons à propos des organisations. Réfléchir à ce contact, voilà qui pourrait passer pour subjectif, c'est pourtant pour Schütz l'étape indispensable pour toute prétention à la validité : «La sauvegarde du point de vue subjectif est la seule garantie (cependant suffisante) que le monde de la réalité sociale ne se verra pas substituer un monde fictif inexistant construit par l'observateur scientifique. » (2007, p. 23). Le danger d'un excès de prise de distance ou de souci d'objectivité est de construire un monde fictif posé pour réel. La prise de distance ne peut venir qu'après une exposition aussi ouverte que possible à l'organisation étudiée, et après avoir laissé cette exposition mettre en mouvement nos présupposés (Moriceau et Soparnot, à paraître). La description témoigne de notre exposition, elle l'inscrit, la partage, elle atteste que nous étions là, dans cette atmosphère, auprès des acteurs et de archives, que nous avons rencontré ce bout de monde dont nous parlons.

L'écriture dit et trahit le contact avec ce monde. Plus qu'une approche elle est une esthétique, une façon de toucher le monde, de s'ouvrir à lui. Elle est notre façon de le percevoir par nos sens, notre sensibilité. C'est cette esthétique, cette sensibilité que restitue l'écriture, qu'est l'écriture. Le travail d'écriture est la recherche de l'expression de ce contact informé. Alors travailler l'écriture, c'est d'abord tenter de restituer le monde tel qu'il nous apparaît et c'est proposer d'autres être-au-monde, voire une symphonie de nouveaux mondes. Solé (2000) y insiste, nous sommes des créateurs de monde et nous créons des mondes avec nos mots. Les écrivants aussi créent le monde, mais en voulant user plutôt que problématiser l'écriture, ils perdent la possibilité de réfléchir à cette construction.

C'est en effet seulement quand nous écrivons que nous nous rendons compte de notre position, de nos

croyances, de notre mode de réflexion, du parcours de notre pensée. Écrire c'est dire «je », «nous » ou « on », c'est choisir certains mots, c'est exposer ses inférences, devoir montrer des sources, etc. D'où écrivonsnous, pour qui, avec quels arguments, en invoquant quelle théorie, quels indices ? Qu'incluons-nous dans la description ? Voici que l'habituel, le tenu pour évident, deviennent questions à examiner, thèmes à débattre et pratiques à discuter. Les bonnes pratiques de la recherche deviennent problématiques. C'est souvent lors d'un tel exercice de réflexivité que nous découvrons quelque chose d'original à raconter, qu'une thèse se forme, qu'un besoin de chercher plus loin, de lire et de réfléchir devient impérieux (LETICHE et LIGHTFOOT, 2014).

Mais voici qu'écrire et décrire, ce que peut-être nous souhaitions neutre au départ, fait apparaître des groupes sous un jour favorable et d'autres défavorable. Nous comprenons que nos écrits contribuent à la construction de leur identité et éventuellement de leur position. Nous nous rendons compte que, volontairement ou non, nos textes ont toujours un impact politique, potentiellement libérateurs ou contribuant à l'oppression. Et nous comprenons que notre façon de revendiquer une autorité un savoir, font aussi parti d'un jeu politique. Le terrain que nous décrivons, le présentons-nous comme illustration, preuve, témoignage, exploration, but de la recherche, engagement? Tout comme nous nous rendons compte que nos affirmations peuvent blesser certains participants, les stigmatiser ou leur assigner une identité, ou encore leur donner attention, pouvoir, qu'elles participent peut-être à soigner une blessure. Le travail d'écriture enclenche la réflexivité :

«Réflexivité (...) signifie qu'une attention sérieuse est portée sur la manière selon laquelle les différents éléments linguistiques, sociaux, politiques et théoriques sont tissés ensemble dans le processus de développement des connaissances, au cours duquel le matériel empirique est construit, interprété et écrit. » (ALVESSON \& SKÖLDBERG, 2000, p. 9)

Voici que la réflexivité dans l'écriture peut se retourner sur le design de la recherche (le choix de la question, des cadres théoriques, des terrains), sur la langue utilisée, sur notre position vis-à-vis du terrain, et sur notre lien avec ce que nous étudions, en référence à nos expériences de vie, notre ethnie, classe, genre, culture, idéologie, etc. Réflexions qui à leur tour vont influencer le travail d'interprétation et d'écriture.

L'écriture est ainsi au cœur d'un travail de recherche authentique, elle n'en est pas seulement son prolongement ou sa mise en valeur. Il est sans doute temps de nous tourner vers des questions plus spécifiques, notamment celles de la représentation, de la voix et de la performativité. 


\section{ÉCRITURE NON REPRÉSENTATIVE}

Problématiser l'écriture qualitative, lors de la description d'un terrain en sciences sociales, ce sont sans doute les ethnographes qui y ont été les premiers et les plus sincèrement confrontés. L'histoire serait longue mais l'un des moments clés a été ce qui a été appelé la crise de la représentation au milieu des années 1980. Dans leur volonté de représenter la culture de l'autre, la conscience était de plus en plus vive que cette entreprise se heurtait à au moins deux difficultés. La première était celle que même en tentant de montrer le point de vue indigène, la description se faisait dans la culture du chercheur, celui étant pris dans ses propres circuits politiques, institutionnels, historiques ou rhétoriques (CLIFFORD, 1986). La description ne pouvait se faire d'une plateforme indépendante de toute culture. Le chercheur devait traduire ici ce qu'il vivait là-bas, transformant leurs vies en son œuvre (GEERTZ, 1988). Et cette transcription était faite par un auteur, dont le style propre était toujours présent, style déjà chargé de théorie (idem). La seconde était que l'ethnologue était mis en position de devoir parler au nom de l'autre, sans que celui-ci n'eut de regard sur cette voix (DENZIN \& LINCOLN, 2005, DENZIN, 2003). La culture n'était alors pas représentée mais bien davantage créée par les textes des ethnologues. Leur rôle ne devait-il pas alors être plutôt d'évoquer, autrement dit de donner à vivre au lecteur l'expérience qu'ils avaient vécue au contact de la culture (TYLER, 1986) ?

On peut prendre ce point de départ pour suivre les innombrables efforts pour échapper aux limites et pièges de la représentation. En empruntant la saisissante formule de ZERNIK (2010) à propos du cinéma, nous pourrions dire que bien des textes, et souvent les plus réussis, tentent non pas que le monde devienne image grâce à la représentation mais parviennent à faire qu'une image, un récit, deviennent monde par une esthétique de la présence. Un portait, une rencontre, une vignette, une histoire, un objet, une parole, une impression, un rythme, nous voici invités à entrer dans un monde, à reconstituer un monde, sans qu'il soit trop tôt pris dans les filets de la représentation, avec ses règles géométriques de la perspective, ses conventions aveugles, son immobilité, ses enjeux de pouvoir à chaque trait. S'appuyant notamment sur le post-structuralisme (Foucault, Deleuze, Derrida, puis Rancière), à la suite des ethnographes, de nombreux auteurs en théorie des organisations ont renoncé aux descriptions habituelles du terrain pour s'aventurer dans de nouveaux domaines, avec leurs possibilités mais aussi avec les questions que celles-ci emportaient avec elles (MORICEAU, 2014).

La représentation transforme en effet le terrain en un champ, que l'on entende par là le champ de conscience, le champ bourdieusien ou le champ balayé par la caméra. On s'inquiète alors de ce qui est laissé hors champ, hors de la visibilité et donc hors de la réflexion. La recherche qualitative a été particulièrement créative quant aux modes d'écriture et de description tentant d'inclure de nouvelles dimensions dans le champ. Elle s'est ainsi élancée dans de nombreux tournants, découvrant chaque fois de nouveaux aspects, jusque là insuffisamment pensés. Le corps, les récits, la matérialité, l'espace, les affects, la pratique, le genre, la mémoire, le territoire, etc., pour être pensés doivent d'abord être convoqués dans la description. Mais une écriture maladroite risque de durcir ces dimensions de l'expérience en variable mesurable ou en cause unique. Il s'agit avant tout de restituer cette expérience, de faire ressentir l'effet de ces dimensions supplémentaires, d'évoquer, de donner à voir et expérimenter, de mettre au premier plan ce qu'on croyait secondaire. L'écriture doit s'armer de nouveaux mots et de nouvelles expressions pour dire par exemple la présence des corps, leurs façons de se montrer et de se cacher, leurs modes de communication et d'influence, comment ils teintent ou contraignent discours, pratiques et décisions, le continent de l'indicible à leur propos, l'érotisme et le tabou, le symbolique et le religieux, le stylé et le conforme, l'intime et le complexé, qu'ils jettent dans la vie organisationnelle. Et voici que les modèles se truffent d'images, de métaphores, de figures, de formes, d'écarts à la syntaxe, de formulations poétiques pour les organisations (Pesqueux, 2002).

Mais questionner la représentation, ce n'est pas seulement ouvrir le champ, c'est aussi s'inquiéter du contre champ. Car si le corporel, l'affectif, le genre, le matériel par exemple sont reconnus comme importants pour les acteurs étudiés, on ne peut ignorer leur influence sur le chercheur et la recherche. Il est difficile de poser les acteurs pris dans des jeux organisationnels, lestés d'un corps, mus par des affects, influencés par des éléments matériels, etc. et supposer que le chercheur et la recherche en seraient exempts. L'écriture en devient plus difficile et plus riche puisqu'elle émane d'un chercheur qui doit prendre en compte ses jeux organisationnels, sa corporéité, etc. pour décrire et penser ce qu'il étudie. Dans le tournant vers les affects, c'est justement parce que le chercheur est affecté qu'il peut être sensible et comprendre les affects en jeu, tout en sachant qu'il affecte aussi à son tour la situation à décrire. Et c'est parce qu'on suppose que le lecteur n'est pas fait d'une étoffe différente qu'il sera affecté et comprendra l'expérience décrite. Avec l'autoethnographie, champ et contre-champ se recouvrent. Le chercheur décrit l'expérience qu'il a vécue en première personne mais également sa réflexivité de chercheur enquêtant sur sa propre expérience. La description cherche alors à « montrer plusieurs niveaux de conscience, connectant le personnel et le culturel » (ELLIS \& BOCHNER, 2000, p.739). Pour reprendre les mots d'ElLIS (2004), il s'agit de penser comme un ethnographe, décrivant le plus précisément possible l'expérience, les faits, les contextes, les pensées et sentiments et écrire comme un romancier pour restituer l'expérience avec toute la force et le suspens d'un moment vécu.

Plus encore, parfois le besoin est exprimé de remplacer la description du champ par l'arpentage d'un paysage, ou d'un territoire. À l'image fixe et fidèle se substitue une collection de récits de contacts avec le 
terrain en différents lieux et différents moments. Donnons deux exemples. Lorsque STEWART (2007) veut donner à penser le capitalisme contemporain, elle dit vouloir ralentir le saut vers la représentation et le jugement pour se laisser affecter par la façon selon laquelle le capitalisme nous touche et s'imprime en nous. Ce qu'elle décrit, ce sont des moments d'affects ordinaires trahissant des forces et des intensités qui passent entre nos corps, nos rêves, nos drames et nos façons de faire monde. Point d'image fixe, ou de moments symboliques, mais une série de vignettes, de scènes touchantes et mouvantes dont la succession laisse une oppressante impression d'imminence et de pression. Point de représentation du capitalisme, mais une présentation de ces manifestations, multiples, obliques, qui se cachent dans le détail du plus quotidien. C'est également une volonté de capter les sensations et les intensités, les rythmes, rencontres, événements et atmosphères du monde organisationnel dans la diversité de leurs manifestations qui président aux approches non-représentationnelles (cf. par exemple BEYES \& STEYAERT, 2011). Mais alors la recherche ne peut seulement décrire, elle participe de ces flux, elle est processus qui s'ajoute aux processus étudiés. Recherche et théories ne représentent pas, elles sont performances. Elles témoignent, et ce faisant elles influencent. L'enquête ne reflète pas, elle produit. L'écriture académique ne décrit pas l'organisation en soi, elle participe à sa production. Représenter reviendrait à figer ce qui est mouvement et en perpétuelle réinvention, la théorie non-représentationnelle accompagne, amplifie ou subvertit cette production.

Ces exemples ou ces expérimentations montrent la place centrale d'une problématisation de l'écriture dans les développements récents des méthodes qualitatives. La description n'y est pas vue selon l'image d'un peintre qui essaierait de représenter ce qu'il perçoit sur un tableau. La critique de la représentation dépasse la question de la perspective et du hors-champ pour la considérer à l'intérieur de dispositifs plus larges. C'est ce que nous fait magistralement comprendre par exemple le commentaire du tableau Las Meninas de Velasquez par Foucault (1966). La représentation des Meninas n'est pas le fruit de la seule expressivité du peintre. Elle est gouvernée par les techniques et conventions picturales, par une place dans l'histoire de la peinture, par la position du peintre par rapport à la royauté, etc. Le dispositif détermine ce qui est visible et ce qui ne l'est pas, le dicible et le pensable, les places de chacun, les manières de se constituer en sujet (DELEUZE, 1986).

Prises en tant que participant d'un dispositif, l'écriture et la description ne pourraient être seulement pensées le long d'une évolution faite de tournants et d'agrandissements à l'image de celle de DENZIN et LINCOLN (2005). La question du style et format de description et d'écriture ne serait pas seulement celle d'un droit au style face à l'austérité d'un supposé langage transparent de la science, ou celle des formats imposés par rapport aux possibilités de compositions plus complexes. Il faudrait interroger les dispositifs dans lesquels ces conventions sont prises, incluant entre autres les modèles économiques des revues, les classements universitaires, les évaluations des chercheurs, la symbolique du quantitatif comme proxy de la scientificité, etc.

\section{LA VOIX ET LES ÉCRITURES PLURIELLES}

Les questions de l'écriture et de la description du qualitatif ne se limitent bien entendu pas au contenu ou au format. Cruciale est la question de la voix. La recherche ne s'écrit pas toute seule, confinée dans l'univers éthéré de la raison pure. Elle s'écrit depuis une position, une manière propre d'envisager les questions, de mener le parcours. La voix définit la relation du chercheur à son objet, au lecteur, au savoir. Elle signe la présence de l'auteur dans son texte et l'adresse du chercheur au lecteur. Si depuis Platon on oppose la voix et l'écriture, la voix est ce qui reste de parole dans une écriture.

Cependant notre voix de chercheur est en général excessivement autoritaire. Nous disons au lecteur quelle part et quelle signification retenir de la littérature, nous sélectionnons les extraits d'entretiens, les observations présentées comme pertinentes, dans quel ordre les présenter, nous expliquons leurs sens, définissons qu'en penser, sélectionnons les auteurs pour les discuter, nous affirmons quelles en sont les implications pour la pratique, etc. Bref, nous maîtrisons l'attention du lecteur de manière à ce qu'il ne puisse échapper à notre argumentation. Ce que Latour, retrouvant la tradition rhétorique, appelle « captation »:

«L'auteur d'un texte scientifique se retrouve ainsi confronté à un dilemme : comment laisser quelqu'un complètement libre et faire en sorte qu'il soit en même temps totalement obéissant ? Quel est le meilleur moyen de résoudre ce paradoxe ? Disposer le texte de façon que, quel que soit l'endroit où le lecteur veuille se rendre, il ne puisse prendre qu'un seul chemin. » (LATOUR, 1989, p. 87- 88).

Si bien des textes académiques sont ennuyeux, la lecture fastidieuse, ce n'est pas seulement parce qu'ils sont mal écrits. C'est aussi du fait qu'ils nous « captent », non au sens d'être captivants, au sens de nous assigner à une place subalterne. Nous ne sommes pas mis au travail. Pas de travail d'interprétation, de travail d'imagination, de travail de réflexion ou de création, pas de réelle énigme, pas de double-entendre, ironie ou second degré, pas de réponse à donner à l'auteur. Dans le partage du sensible, l'auteur maîtrise toute la création du sens. Il use de son autorité pour affirmer ce dont le lecteur doit être persuadé. BARTHES (1970), au contraire, montre l'intérêt de mettre le lecteur au travail, afin de construire en commun, dans un processus jamais achevé, de la pensée. Si on suit sa proposition, il n'y a pas d'un côté celui qui sait et de l'autre celui qui ne sait pas encore, mais deux partenaires du dialogue, complices pour créer de la réflexion. En montrant un ensemble de 
faits, en relayant une atmosphère, des processus, en donnant une série d'indices et de signes, le lecteur ressent, il construit sa propre interprétation et comprend d'autant mieux qu'il aura été actif dans cette élaboration. Ce n'est pas que l'auteur doit s'abstenir de proposer toute théorie, au contraire, mais alors celle-ci sera plus proposée qu'imposée, appelant moins obédience que discussion. Le lecteur l'accueillera, à armes presque égales, et pourra juger le travail interprétatif proposé.

C'est à une autre forme de capture que l'écriture féminine tend à résister. La forme autoritaire n'est qu'un aspect du primat dans les théories des organisations d'une écriture masculine : dominatrice, rationnelle, pénétrante, patriarcale (PULLEN \& RHODES, 2015) - phallogocentrique ${ }^{2}$. La recherche et la théorie sont produites par un corps, l'écriture féminine va tenter de retrouver cette origine incarnée, de capter l'expérience, l'affect, le rythme avant qu'ils ne soient ordonnés dans le symbolique (HöPFL, 2000). Ce contact plus originaire, ou autre que viril, sera exprimé par des formes poétiques, subversives, joueuses, incarnées, autoethnographiques, artistes, évitant les catégorisations rationnelles, les identités fixées, le monopole du rationnel, de l'abstrait, de l'impersonnel. Souvent, pour éviter d'être marginalisées, ces écritures mélangent et jouent entre le rationnel et l'impénétrable, le poétique et le formel, le conforme et le subversif à la recherche d'autres voix, d'autres dicibilités, visibilités, identités, expériences. D'autres formes de savoir, de comprendre et de communication. Le féminin, ou le queer, ne se pose généralement pas en opposition avec le masculin (PHILLIPS et al., 2014), mais cherche la possibilité à être autre, de multiples façons d'être autres, à inventer de nouvelles formes, de nouvelles pratiques, de nouvelles voix.

La voix du chercheur est également en général ethnocentrée. Même lorsqu'on veut comprendre l'autre, les concepts (et la langue) sont ceux de l'occident. On en peut éviter de voir là une résistance du colonial. C'est ce que dénonçait déjà SAÏD (1978) : même lorsqu'elle s'intéresse à l'orient, l'orient de l'académie est l'orient de l'occident. Les savoirs, les concepts, les méthodes, les modes d'argumentation occidentaux sont supposés supérieurs à ceux des autres cultures (PRASAD, 2003). Un texte qui s'appuierait sur la théorie postcoloniale pourrait même d'une certaine façon être encore colonial tant le concept (et une partie des théories) de postcolonial s'inscrit dans l'histoire et la conception occidentale (WESTON \& IMAS, 2018). L'écriture postcoloniale tente d'échapper à ce colonialisme par deux voies principales. D'abord en incorporant et se fondant sur des savoirs, concepts et modes d'expression «indigènes ». Ensuite en travaillant avec des représentants de l'autre culture pour la conception de la recherche et l'interprétation. On cherchera à ce que les interlocuteurs ou participants s'expriment dans leurs propres modalités culturelles, par exemple sous forme de performances (DENZIN, 2003). On pourra tenter, pour reprendre une expression de Deleuze et Guattari, de faire un usage mineur de la langue académique majeure (MORICEAU, 2017). L'écriture acoloniale tentera de renverser et déchirer ces oppositions. Écriture de la pensée du tremblement, du tout-monde (GLISSANT, 1997), de la créolité, autant d'écritures de l'éclatement des démarcations et des étiquettes. Écritures qui demandent de substituer aux critères habituels de validité (eurocentrés) un travail de réflexivité notamment sur les différents ancrages culturels et sur les modes d'écriture (WESTON \& IMAS, 2018)... tout en essayant de se faire publier.

Toutefois, la situation du peuple lointain est-elle si différente de l'autre plus proche ? La voix du chercheur ou de la chercheuse reste la voix maîtresse, intégrant et ainsi soumettant toutes les autres voix dans son propre discours. La voix de l'autre est ainsi prise dans ses cadres, dans ses catégories. Ce qui est énoncé est ainsi recontextualisé dans un récit plus large qui est sensé en montrer la signification plus profonde. C'est par exemple ce que dénonce Rancière, comme le montrent magistralement MARQUES et OLIVEIRA dans ce numéro. La voix de l'autre est accueillie comme expression d'une condition, description de première main d'une situation. Mais la théorisation, le dernier mot interprétatif reste le monopole du chercheur. Rancière au contraire pose une égalité de principe des intelligences et voudrait donner à la prise de parole du peuple une égale place dans la scène des mésententes.

Mais ces questions lestent aussi nos descriptions. Comment faire pour que la voix qui préside à la description soit composée des voix des différents participants, avec leurs langages propres ? Que tout ne soit pas aligné dans une logique mais que la description même comporte plusieurs logiques dont certaines se combattent ? C'est ce que Bakhtine cherchait dans le roman avec la polyphonie, l'hétéroglossie et le dialogisme. Il y a une pluralité de voix qui se concurrencent pour être entendues dans les organisations, on apprend beaucoup à les repérer et les écouter mais ne reproduit-on pas ce qu'on dénonce par une écriture monophonique et monologique (cf. BELOVA, KING, SLIWA, 2008) ? Citer des verbatims ne suffit pas à faire polyphonie car la voix du chercheur les englobe, car une langue maîtrise toutes les autres, celle de la recherche, car une logique traverse et préside à toutes les autres, celle de la démonstration. Faut-il alors partager l'écriture avec les participants ou inventer des formes polyphoniques et dialogiques d'écriture ? Et respecte-t-on l'altérité de la voix de l'autre en l'incluant dans un texte académique (LETICHE, 2010)?

\footnotetext{
${ }^{2}$ Expression forgée par DERRIDA (1966) pour désigner une auto-institution à partir d'une parole masculine, autoritaire et rationnelle - à la manière d'une chose qui se présente comme « Moi, la vérité, je parle » (selon une expression de Lacan).
} 


\section{ÉCRITURES PERFORMATIVES}

Si l'on cherche à avoir une voix, à prendre la parole, c'est avec l'espoir d'avoir un effet. L'écriture n'est pas simplement un reflet, un constat; elle ne fait pas que dire le monde, elle agit sur lui. C'est ce qu'AUSTIN (1962) appelle la performativité. Le texte est adressé aux lecteurs, et au-delà d'eux à tout un réseau de pensées, d'actions et d'effets à venir. Il ne fait pas que véhiculer un sens, il joue sur le sensible, le sensitif, les sensations.

«Ce n'est pas en décrivant que les mots accomplissent leur puissance : c'est en nommant, en appelant, en commandant, en intrigant, en séduisant qu'ils tranchent dans la naturalité des existences, mettent des humains en route, les séparent et les unissent en communautés. Le mot a bien d'autres choses à imiter que son sens ou son référent : la puissance de la parole qui le porte à l'existence, le mouvement de la vie, le geste d'une adresse, l'effet qu'il anticipe, le destinataire dont il mime par avance l'écoute ou la lecture. » (RANCIÈRE, 1998, p.11)

Les chercheurs en gestion voudraient avoir un «impact», que leurs théories soient utilisées par les acteurs pour décider, diriger, communiquer (par exemple CABANTOUS \& GOND, 2011). Les mots alors peuvent se doubler de dispositifs sociotechniques pour renforcer ou imposer cette influence (CALLON \& MUNIESA, 2005). Après tout, n'est-ce pas le but d'une certaine recherche en gestion que d'orienter les comportements organisationnels ? Pourtant, les effets des écrits académiques sont certainement plus larges que leurs éventuelles applications à la pratique (GOND et al., 2016 ; MORICEAU, 2016). Ils classent, catégorisent, désignent, donnent à voir, font ressentir, forcent à penser. Ils participent à la construction des identités individuelles, professionnelles et sociales, chacun se définissant en relation (se conformant, se rebellant, subvertissant) avec les discours à son propos (BUTLER, 1997). Ils définissent des places, des légitimités de parler, des visibilités de certains acteurs, des problèmes considérés comme légitimes et prioritaires par rapport à d'autres subalternes (RANCIÈRE, 2000). Les effets dépendent des résonances avec d'autres textes, leurs reprises dans d'autres circuits discursifs, d'autres contextes, d'autres supports, leurs traductions et parasitages, etc. (DERRIDA, 1972).

Ainsi par exemple, bien des descriptions ne vont pas faire que dépeindre une organisation, elles vont provoquer, souvent intentionnellement, des affects chez le lecteur. Par exemple, dans l'extrait suivant, la description vise probablement à provoquer un certain dégoût et une révolte de la part du lecteur, afin de l'amener à envisager qu'une action en apparence généreuse de RSE peut se révéler un cadeau empoisonné. En emmenant le lecteur dans la décharge, où les «dons » d'ordinateurs vont finir bien trop sûrement et bien trop vite, GUERILLOT (2012, p. 107) veut transformer les images et les discours, convoquant d'autres imaginaires et d'autres jeux de langage :

« Nous arpentons et descendons des reliefs formés par les déchets en faisant attention où l'on met les pieds pour ne surtout pas se blesser ou trébucher. L'odeur empirée à la fois par la chaleur du soleil, par les fumées et par cette file immense de camions à l'arrêt, bloqués par les inondations de la récente saison des pluies augmentant ainsi l'humidité dans l'air, donnent l'impression d'être au milieu d'un no man's land, d'un monde dévasté, chaotique, défriché, abimé, hanté par la mort. Je suis au centre du monde et nulle part en même temps... Cette décharge symbolise violemment le revers de la médaille de ce monde en perdition. »

De même la description peut tenter de troubler ou déstabiliser le lecteur pour l'emmener à réviser ce qu'il croyait savoir avec certitude. Les mêmes mots, mêlés de contraintes et d'enthousiasme, qui semblent montrer l'emprise et l'exploitation capitalistes servent dans l'extrait suivant à montrer les efforts pour justement desserrer cette emprise. Le texte ne fait pas que décrire une expérience. Euvre d'une chercheuse actrice, il est performance. Non seulement le lecteur/spectateur est invité à questionner ses convictions mais également à se demander jusqu'où il est lui-même prisonnier de cette emprise : a-t-il encore du désir, est-il prêt aux efforts pour s'individuer?

« Je me rappelle avoir les cheveux trempés de sueur, du mal à reprendre mon souffle, ne pas écouter les douleurs dans mes membres et reprendre encore plusieurs heures les entraînements sur une musique endiablée, nous portant dans une sorte de transe. De devoir ensuite organiser les actions du lendemain, et devoir faire, sans possibilité d'excuses, les tâches ménagères allouées. Et avant de me coucher, quand d'autres dormaient déjà, m'entraîner longuement à marcher avec des talons trop hauts pour savoir parfaitement tenir mon rôle dans le spectacle à venir. Puis, le lendemain matin être à l'heure pour la nouvelle vague d'entraînement du jour. On pourrait y lire une dérive sectaire, ou l'emprise d'une organisation sadomasochiste. Mais le plus surprenant est que tous paraissaient en être satisfaits et semblaient en tirer, paradoxalement, une énergie qu'ils n'avaient pas connue auparavant. Cette discipline de vie, cette vie en exercice, semblait la voie difficile mais assurée d'aller au-delà de ce qu'on savait faire, la possibilité d'obtenir de soi une certaine transformation. Aller au-delà de ses premières limites était même un chemin de connaissance de soi. » (PAES, à paraître) 
LINSTEAD (2017) encourage de tels textes dont la performativité repose sur une utilisation critique des affects et des procédés artistiques. Ils sont capables d'emporter leur audience vers de nouvelles sensibilités, des apprentissages, des prises de consciences. Il décrit cette prise d'effet sur l'audience (le texte peut se doubler d'images, de musiques, de théâtralité) en quatre moments. D'abord un moment de sidération, esthétique, au cours duquel les habitudes de pensées, les théories normales se bloquent, incapables de donner sens à ce qui se présente. Remplacé très vite par un moment de grande productivité réflexive, poétique, où le lecteur spectateur travaille pour reconstruire du sens possible, liant ce qu'il reçoit à d'autres expériences, concepts et tentatives de faire sens. Progressivement viendra le moment éthique, où ce contact immédiat et affectif avec l'expérience de l'autre l'amène à réfléchir sur ses positions, son action, à s'inquiéter de la condition ainsi présentée, du futur des personnes exposées. Enfin, arrive le moment de critique, politique, où se dessinent pour nous de façon plus visibles, audibles et touchables les différences de pouvoir, les mécanismes d'oppression, les conflits, les effets des changements. La performativité ne vient pas d'une liste d'arguments, elle est le fruit d'une mise en relation, d'un travail du lecteur, d'une incorporation de l'expérience.

Mais la performativité de nos textes n'est pas alors que signe d'une puissance, elle est aussi inquiétude et responsabilité. Le pouvoir de toucher, de catégoriser, d'affecter demande de notre part une certaine éthique, une responsabilité d'auteur. Nos descriptions et catégorisations accentuent-elles les blessures ou contribuentelles à donner du pouvoir d'agir aux personnes stigmatisées (BOCHNER, 2000) ? Comment parler d'un « terrain sensible », quand nos révélations ou analyses peuvent mettre en danger des personnes ou des positions, accrôitre des vulnérabilités, heurter des sensibilités (HENNEQUIN, 2012) ? Ne faut-il pas se donner le temps long de la maturation, même pris dans le jeu de la course à la publication, quand nous traitons de sujets qui peuvent avoir un impact sur la santé ou les politiques publiques (STENGERS, 2016) ? La conscience d'être dans un monde vulnérable (TRONTO, 2009), qu'il faut savoir parfois porter une voix différente sur les échelles de mesures trop sûres d'elles-mêmes (GILLIGAN, 2008), nous demande de porter, attention, soin, sollicitude à cette relation avec les effets de nos textes. Écrire, n'est-ce pas avant tout communiquer, être en relation, et pas seulement énoncer une vérité ou le résultat d'une mesure - toujours sujette, ne l'oublions pas, à discussion?

La performativité de l'écriture ouvre même les possibilités d'une écriture thérapeutique. La thérapie peut être pour l'auteur. Écrire pour partager une douleur ou témoigner d'une condition, tout e contribuant à la connaissance, peut être l'un des buts de l'autoethnographie par exemple. Écrire à propos du harcèlement moral et du proxénétisme académique à l'université (VALENTIM, 2018) ou d'une migration (WRIGHT, 2008) peut soulager l'auteur tout en nous permettant de comprendre un peu mieux la condition contemporaine. Mais la thérapie peut être également pour le lecteur ou la société. ELLIS et al. (2010) donne l'exemple des écrits de Friedan sur le mécontentement ressenti par de nombreuses femmes américaines de ne pouvoir s'épanouir au travail mais dont aucune n'osait parler. Le texte a pu aider certaines femmes et a inspiré certaines politiques publiques. Enfin les textes sur les organisations, tout comme les textes émanant des organisations, peuvent être examinés comme révélant des symptômes du management et des organisations (BUTLER, 2008), comme autant de portraits cliniques des organisations contemporaines.

\section{CONCLUSION}

La description et l'écriture qualitatives ne sont pas les simples mises en mot de ce qui a été vu et pensé auparavant. C'est une part intégrante de l'enquête, une recherche au sens fort où nous commençons à écrire sans encore savoir où cela va nous mener. C'est un travail de réflexivité, une résistance à la prolétarisation décrite par Stiegler (LETICHE et al., 2017). L'écriture blanche, supposée neutre, du non-style se soumet à un dispositif que l'auteur ne soupçonne souvent même pas, surtout qu'il ne veut pas voir. Apparaissent à la description et à l'écriture bien des questions et des tensions, des enjeux qui définissent la nature et la portée même de notre recherche. S'y révèle le politique de notre recherche (l'autorité, le colonial, le genre, les effets de subjectivation, les partages du sensibles, etc.). S'y révèlent bien des aspects éthiques (les effets performatifs sur les identités, les vulnérabilités, les sensibilités, l'adresse au lecteur, l'ouverture à l'autre, etc.). L'écriture demande de ce fait réflexivité et engagement.

La supposée communication académique de raison à raison ostracise le sensible, le corps, le situé, l'expérience esthétique et subjective, la performativité, la sollicitude, le féminin, le subalterne, le mineur, le plurivoque... elle s'interdit tout un pan tant de contenu que de possibilités de communication. Nous avons vu les possibilités pourtant de l'évocation d'un savoir d'expérience, incorporé, dense, aux multiples possibilités d'interprétation. Les possibilités de textes affectifs et affectant, individuant, enracinés dans les cultures, misant sur les ressources du désir et de l'esthétique, collaborant avec les participants et les lecteurs. Les problèmes organisationnels sont complexes et pluriels, ils ont besoin d'une écriture riche et dense, de toutes les ressources du langage, pour être pensés, pour être gérés.

L'écriture est notre art, notre lame, notre flamme. Veillons à ce savoir-faire. Transmettons-le. Développons de nouvelles écritures qualitatives. Et continuons à écrire comme si écrire pouvait faire une différence (LETICHE \& LIGHTFOOT, 2014). 



\section{BIBLIOGRAPHIE}

Alvesson M. \& SKÖLDBERG K. (2000), Reflexive Methodology: New Vistas For Qualitative Research, London: Sage Publications.

Austin J.L. (1962), How to Do Things With Words, Oxford, Clarendon Press.

BARTHES R. (1970), S/Z, Paris, Seuil.

Belova O., King I. \& Sliwa M. (2008), "Introduction: Polyphony and Organization Studies: Mikhail Bakhtin and Beyond", Organization Studies, Vol. 29, n 4, pp. 493-500.

BEYES T. \& STEYAERT C. (2012), "Spacing organization: Non-representational theorizing and the spatial turn in organizational research”, Organization, Vol. 19, n¹, pp. 45-61.

BuTLER J. (1997), Excitable Speech. A Politics of the Performative. New York: Routledge.

ButLer N. (2008), "Critical and Clinical Management Studies”, Ephemera, Vol. 8, n¹, pp. 7-25.

CABAntous L. \& GOND J.-P. (2011), "Rational decision-making as a 'performative praxis': Explaining rationality's eternel retour”, Organization Science, Vol. 22, n³, pp. 573-586.

CALLON M. \& MuniESA F. (2005), Economic markets as calculative collective devices", Organization Studies, Vol. 26, n8, pp. 1229-1250.

Clifford J. (1986), “Introduction: Partial Truths", in Clifford \& Marcus, Writing Culture: The Poetics and Politics of Ethnography, Berkeley: University of California Press, pp. 1-26.

DANE E. (2011), "Changing the Tune of Academic Writing: Muting Cognitive Entrenchment", Journal of Management Inquiry, vol. 20, $\mathrm{n}^{\circ} 3$, pp. 332-336.

DeleuZE G. (1986), Foucault, Paris: Éditions de Minuit.

DERRIDA J. (1966), De la grammatologie, Paris: Éditions de Minuit.

DERRIDA J. (1972), "Signature événement contexte", in Marges de la Philosophie, Paris: Éditions de Minuit, pp.367-393.

DENZIN N.K. (2003), Performance Ethnography: The Politics and Pedagogies of Culture, London: Sage

DENZIN NK. \& LinCOLN Y. (2005), The Sage Handbook of Qualitative Research, $3^{\text {rd }}$ ed., Thousand Oaks, CA: Sage Publications.

DUMEZ, H. (2010), «La description : point aveugle de la recherche qualitative », Le Libellio d'AEGIS, Vol. 6, ${ }^{\circ}$ 2, pp. 28-43.

ELLIS C. (2004), The Ethnographic I: A Methodological Novel about Autoethnography, Walnut Creek, CA: Altamira Press.

ELLIS C. \& Bochner A.P. (2000), "Autoethnography, personal narrative, reflexivity: Researcher as subject”, in Denzin \& Lincoln (eds.), Handbook of qualitative research (2nd ed., pp.733-769). Thousand Oaks, CA: Sage.

Ellis C, ADAMs T. \& Bochner A. (2010), Autoethnography: An Overview. Forum Qualitative Sozialforschung / Forum: Qualitative Social Research, Vol. 12, n ${ }^{\circ}$, Art. 10.

FoucAult M. (1986), Les Mots et les choses, Paris: Gallimard.

GEERTZ C. (1988), Works and Lives: The Anthropologist as Author. Stanford: Stanford University Press.

GILligan C. (2008), Une voie différente, Paris : Flammarion.

Glissant E. (1997), Traité du Tout-Monde, Paris : Gallimard.

Gond J.-P., CABAntous L., Harding N. \& Learmonth M. (2016), "What do we mean by performativity in organizational and management theory? The uses and abuses of performativity", International journal of management reviews, Vol. $18, \mathrm{n}^{\circ} 4$, pp. 440-463.

GUÉRILLOT G. (2012), La réception des discours de développement durable et d'actions de responsabilité sociale des entreprises dans les pays du Sud. Le cas d'un don d'ordinateurs au Sénégal dans le cadre d'un projet tripartite de solidarité numérique, thèse soutenue à Télécom Ecole de Management en collaboration avec l'Université d'Evry Val d'Essonnes, Evry, 05 Décembre.

HENNEQUIN E. (2012), La recherche à l'épreuve des terrains sensibles : Approches en sciences sociales, Paris : L'Harmattan.

HöPF, H. (2000), The suffering mother and the miserable son: Organizing women and organizing women's writing, Gender, Work \& Organization, Vol. 7, n², pp. 98-105.

LAMBERT F. (2007), L'écriture en recherche, Paris: ParcourSic.

LAROCHE, H. (2017), « Mal écrire pour bien publier. À propos de Learn to Write Badly. How to Succeed in the Social Sciences", Le Libellio d'AEGIS, Vol. 13, n 4 - Hiver 2017, pp. 13-30.

LATOUR B. (1989), La science en action, Paris, La Découverte.

LETICHE H. (2010), Polyphony and its Other, Organization Studies, Vol. 31, n³, pp. 261-277.

Letiche H. \& Lightfoot G. (2014), The Relevant PhD, Rotterdam: Sense Publisher.

LETICHE H., LightFOOT G. \& LiLLEY S. (2017), “Classements, capitalisme académique et affects des chercheurs en gestion », Revue Française de Gestion, n²67, pp. 97-115.

LINSTEAD S. (2017), "Feeling the Reel of the Real: Framing the Play of Critically Affective Organizational Research between Art and the Everyday", Organization Studies, published online 03/08/2017. 
MARTinet, A. (2006), Stratégie et pensée complexe. Revue française de gestion, $\mathrm{n}^{\mathrm{o}}$ 160, (1), 31-45.

MORICEAU J-L. (2014) "Tout contre la représentation. Et si le but de la recherche n'était pas de représenter ? ", in Société de Philosophie des Sciences des Gestion (dir), Les Prêts-à-penser en épistémologie des sciences de gestion, Paris : L'Harmattan, pp. 61-79.

MoriCEAU J-L. (2016), "The Turn to Performativity and the Democratic Concern: Four Orientations for a more Demos-Sensitive Debate", in Letiche, Lightfoot \& Moriceau, Demo(s); Philosophy - Pedagogy - Politics, Rotterdam: Sense Publishers, pp. 167-179.

MORICEAU J-L. (2017), "I thought I only had to have an idea", M@n@gement, Unplugged - Academic nonfiction, Vol. 20, n³, pp. 298-299.

MORICEAU J-L. \& SOPARNOT R. (à paraître), S'exposer, Cheminer, Réfléchir : Composer sa méthode de recherche qualitative, Cormelles le Royal, EMS.

PAES I. (à paraître), "Le sens au carrefour des individuations : la construction du performer », in Moriceau, Letiche \& Le Theule, L'Art du sens dans les organisations, Laval : Presses de l'Université Laval.

PESQUEUX Y. (2002), Organisations: Modèles et représentations, Paris : Presses Universitaires de France.

Phillips M., PUllen A. \& RhOdES C. (2014), "Writing Organization as Gendered Practice: Interrupting the Libidinal Economy”, Organization Studies, Vol. 35, n³, pp. 313-333.

PRASAD A. (ed.) (2003), Postcolonial Theory and Organizational Analysis: A critical Engagement. New York: Palgrave.

PUllen A. \& RHODES C. (2015), Writing, the Feminine and Organization, Gender, Work and Organization, Vol. 22, n 2, pp. 87-93.

RANCIÈRE J. (1998), La Chair des mots, Paris : Galilée.

RANCIÈRE J. (2000), Le Partage du sensible, Paris : La Fabrique.

RICHARDSON, L. (2000), «Writing: A mode of Inquiry », in N. Denzin \& Y. Lincoln (Eds), Handbook of Qualitative Research, 2ed (pp. 923-948), Thousand Oaks, CA: Sage Publications.

SAÏD, E.W. (1978), Orientalism. New York: Vintage Books.

SCHÜTZ A. (2007), Essais sur le monde ordinaire. Paris, Le Félin/Kiron (Le Félin poche).

SOLÉ A. (2000). Créateurs de monde. Nos possibles, nos impossibles, Monaco : Editions du Rocher.

STENGERS, I. (2016), “Another science is possible!' A plea for slow science”, in Letiche, Lightfoot, Moriceau, Demo(s): Philosophy, Pedagogy, Politics, Sense Publisher, pp.53-70.

STEWART K. (2007), Ordinary Affects, Durham: Duke University Press.

SwORD H. (2012), Stylish Academic Writing, Cambridge: Harvard University Press.

Tronto J. (2009), Un Monde vulnérable. Pour une politique du care, Paris: La Découverte.

TYLER S. A. (1986), «Post-Modern Ethnography : From Document of the Occult to Occult Document, in Clifford \& G. Marcus, Writing Culture: The Poetics and Politics of Ethnography, Berkeley: University of California Press, pp. 122-140.

VALENTIM I.V.L. (2018), "Between Academic Pimping and Moral Harassment in Higher Education: an Autoethnography in a Brazilian Public University", Journal of Academic Ethics, Online First Articles.

VAN MAANEN J. (1995), "Style as theory", Organization Science, Vol. 6, n²1, pp.133-142.

VAN MAANEN J. (2011), Tales of the Field. On writing ethnography, $2^{\text {nd }}$ ed., Chicago: The University of Chicago Press.

WEICK K.E. (1995), Sensemaking in organization, Thousand Oaks, CA, Sage Publications.

WeStON A. \& IMAS M. (2018), "Resisting Colonization in Business and Management Studies: From Postcolonialism to Decolonization", in Cassel, Cunliffe \& Grandy, The Sage Handbook of Qualitative Business and Management Methods, London: Sage Publications, pp. 119-136.

Wright J.K. (2009), “Autoethnography and Therapy Writing on the Move”, Qualitative Inquiry, Vol. 15, $\mathrm{n}^{\circ} 4$, pp. $623-640$.

ZERNIK C. (2010), Perception-cinéma. Les enjeux stylistiques d'un dispositif, Paris, Vrin. 